\title{
Validación de la sexta edición del Addiction Severity Index (ASI-6) (ISA-6) en población clínica de Colombia
}

Juan Pablo Zapata ${ }^{1}$, Jenny García ${ }^{1,2}$, César Augusto Arroyave ${ }^{2}$, Juan Daniel Calderón², José Mario Gómez ${ }^{3}$, Doris Jacqueline Buitrago ${ }^{3}$, Angélica Aparicio ${ }^{4}$, Daniel Camilo Aguirre ${ }^{1}$

${ }^{1}$ Grupo Académico de Epidemiología Clínica, GRAEPIC, Facultad de Medicina, Universidad de Antioquia, Medellín, Colombia

${ }^{2}$ Clínica Samein S.A.S., Medellín, Colombia

${ }^{3}$ Centro de Atención a Drogodependencias, Hospital Universitario San Vicente Fundación, Medellín, Colombia

${ }^{4}$ Corporación Cardynal y Fundación Camino a la Vida, Medellín, Colombia

Introducción. Se recomienda el uso del Addicition Severity Index en su sexta versión (ASI6) para establecer las necesidades del paciente con adicciones y evaluar la efectividad de los programas.

Objetivo. Desarrollar una versión para Colombia del ASI-6 garantizando la equivalencia translingüística y transcultural, y evaluar su confiabilidad y la validez de constructo. Materiales y métodos. En el estudio se incluyeron adultos con trastornos debidos al uso de sustancias bajo tratamiento en centros de atención de la drogodependencia. Se adaptó la versión en español con base en la original en inglés y, posteriormente, se tradujo al inglés la versión así adaptada para determinar su equivalencia. Se evaluó la confiabilidad en cuanto a la consistencia interna, así como la reproducibilidad prueba-reprueba y la confiabilidad interevaluador. La validez convergente del constructo se comprobó mediante la correlación con cuestionarios que miden constructos similares a los subyacentes a cada subescala del ISA-6. Resultados. Se encontró una consistencia interna adecuada, con un alfa de Cronbach por encima de 0,7 en todas las subescalas, excepto la "Social" $(a=0,66)$. La confiabilidad entre evaluadores y de la prueba-reprueba fueron altas, con coeficientes de correlación intraclase mayores de 0,7 en todas las subescalas. El aspecto convergente de la validez de constructo se demostró al obtener coeficientes de correlación de Spearman entre 0,53 y 0,88 en las subescalas del ISA-6 comparadas con cuestionarios que miden constructos similares. Conclusión. La versión del ISA-6 adaptada al contexto colombiano evidenció una confiabilidad y una validez de constructo adecuadas, por lo que puede introducirse en la clínica, aunque se requieren estudios para conocer su sensibilidad al cambio y su validez estructural.

Recibido: 08/06/18

Publicado: $17 / 10 / 18$

Citación:

Zapata JP, García J, Arroyave CA, Calderón JD, Gómez JM, Buitrago DJ, Aparicio A, Aguirre DC. Validación de la sexta edición del Addiction Severity Index (ISA-6) en población clínica de Colombia. Biomédica. 2019;39:385-404.

https://doi.org/10.7705/biomedica.v39i3.4536

\section{Correspondencia:}

Juan Pablo Zapata, Facultad de Medicina, Universidad de Antioquia, Carrera 51d N 62-29, Medellín, Colombia

Teléfono: (574) 219 6060; fax: (574) 2638282

juanp.zapata@udea.edu.co

\section{Contribución de los autores:}

Juan Pablo Zapata, Jenny García, Daniel Camilo Aguirre: concepción y diseño de la investigación y análisis de datos

Juan Pablo Zapata, Jenny García, César Augusto Arroyave, Doris Jacqueline Buitrago: adaptación cultural del instrumento y evaluación de pacientes Juan Daniel Calderón y Angélica Aparicio: evaluación de pacientes

José Mario Gómez: adaptación cultural del instrumento

Todos los autores participaron en la escritura del manuscrito.

Financiación:

Este trabajo fue financiado por el Grupo Académico de Epidemiología Clínica (GRAEPIC) y la Clínica Samein S.A.S

\section{Conflicto de intereses:}

Los autores declaran no tener conflicto de intereses.
Palabras clave: trastornos relacionados con sustancias; drogas ilícitas; consumidores de drogas; estudios de validación; psicometría.

\section{Validation of the sixth version of the Addiction Severity Index (ASI-6) for patients in a clinical population of Colombia}

Introduction: The Addiction Severity Index (ASI-6) is recommended for identifying the needs of addicted patients and assessing the effectiveness of a program.

Objective: To develop a version of the ASI-6 suitable for Colombia and trans-linguistically and trans-culturally equivalent to the original. Additionally, this study also sought to assess the reliability and construct validity of the resulting version.

Materials and methods: The study included Colombian adults with harmful substance use or dependence syndrome who were being treated at drug addiction centers. The original English version underwent a cultural adaptation process. The scale was translated and back-translated to assess its equivalence. Reliability was assessed in terms of internal consistency and interrater and test-retest reliability. The convergent aspect of the construct validity was assessed via the correlation of the instrument with other scales measuring similar underlying constructs. Results: We found an adequate internal consistency for the subscales of the ASI-6 for its Cronbach's alpha coefficient was above 0.7 with the exception of the social problems subscale $(a=0.66)$. In addition, interrater and test-retest reliability was high, since their intraclass correlation coefficient (ICC) was above 0.7 for all the subscales. Construct validity was demonstrated by a Spearman correlation coefficient ranging from 0.53 to 0.88 between the ASI-6 subscales and other similar scales.

Conclusion: The version of the ASI-6 adapted to the Colombian context was found to have good reliability and validity, thus it can be introduced into clinical practice. However, additional studies are needed to evaluate its responsiveness and structural validity.

Keywords: Substance-related disorders; street drugs; drug users; validation studies; psychometrics. 
La adicción a sustancias es un trastorno mental complejo que se caracteriza por el consumo compulsivo de drogas a pesar de los esfuerzos por abstenerse y de las consecuencias negativas que tiene en el individuo y en su entorno (1).

Según el Informe Mundial sobre las Drogas 2017, publicado por la Oficina de las Naciones Unidas contra la Droga y el Delito (UNODC), se estima que 250 millones de personas entre los 15 y los 64 años consumieron drogas ilícitas en el 2015, lo que corresponde a una prevalencia anual global de 5,3\%. De ellos, cerca de 29,5 millones presentaban un trastorno por el uso de sustancias, o dependencia, lo cual equivaldría a una prevalencia global de 0,6 \% (2).

Según los datos del Estudio Nacional de Consumo de Sustancias Psicoactivas 2013, en Colombia la prevalencia del consumo de cualquier sustancia ilícita durante el año anterior en personas entre los 12 y los 65 años fue de 3,6 \%, siendo la mayor la del grupo de 18 a 24 años (3). Se estimó que en el $57,7 \%$ de los consumidores el uso de las sustancias era perjudicial, lo que indica que por lo menos uno de cada dos consumidores en Colombia presentaba algún problema derivado del consumo. Los departamentos con la mayor prevalencia fueron Antioquia (especialmente Medellín y el Área Metropolitana), Quindío y Risaralda.

El impacto generado por la adicción va más allá del terreno médico, lo que explica que se la considere un problema social y político (4). En el individuo se producen alteraciones en su salud física y mental que muy posiblemente aumentan la demanda y los costos de los servicios de salud (5). También se presenta un aumento en la mortalidad que, junto con la morbilidad, implican una carga alta de enfermedad para la sociedad; de hecho, es una de las primeras cinco causas de años de vida ajustados por discapacidad (6). Asimismo, la adicción ha sido relacionada con múltiples acciones ilegales, como la delincuencia y el crimen organizado (7). Además, en las familias de los individuos con adicción hay un mayor riesgo de violencia intrafamiliar y maltrato (8). Es decir, la adicción repercute en el individuo y en el colectivo, de ahí que se considere una prioridad en salud pública.

La actual estrategia mundial de la UNODC para promover políticas de tratamiento éticas y basadas en la evidencia recibe el nombre de Treatnet (9), y con ella se promueve la individualización del tratamiento en función de las necesidades del sujeto. Para lograrlo, se recomienda el uso sistemático del Addiction Severity Index (ASI), el cual evalúa las diferentes dimensiones que resultan afectadas por el trastorno (10). La versión más reciente es la sexta, la cual ofrece la ventaja de reducir el tiempo de aplicación, pues en el inicio de cada área se incluyen preguntas de tamización (11). Esta versión ya ha sido validada en España y Brasil y se ha encontrado que tiene buenas propiedades psicométricas $(12,13)$.

En Colombia se ha utilizado la primera versión de este instrumento en diferentes instituciones desde 1985 tras un proceso de adaptación realizado en el Hospital Mental de Antioquia en el que no se evaluaron sus propiedades psicométricas (14), lo cual es fundamental dada la influencia del medio cultural en la aplicación de la escala. En las adicciones, la comprensión de algunas conductas y la interpretación de la consecuencias derivadas del consumo deben hacerse a la luz de la cultura en la que se inscriben (15). Asimismo, la formulación de las preguntas y la denominación de las sustancias pueden afectar la comprensión de la escala por parte del examinado y llegar a producir errores de medición (16). Un instrumento confiable y válido es esencial para caracterizar a los pacientes que consultan. 
El objetivo de este estudio fue desarrollar una versión para Colombia de la sexta edición del ASI (ASI-6) que tuviera equivalencia translingüística y transcultural con respecto a la original, así como evaluar la confiabilidad y la validez de constructo en pacientes adultos con diagnóstico de adicción atendidos en centros de atención de las drogodependencias.

\section{Materiales y métodos}

Se hizo un estudio multicéntrico de validación del cuestionario, el cual se ajustó a las disposiciones de la Resolución 008430 de 1993 del Ministerio de Salud de Colombia y de la Declaración de Helsinki de 2013 y fue aprobado por los comités de ética de la Facultad de Medicina de la Universidad de Antioquia y de las instituciones participantes.

Se obtuvo el consentimiento informado de todos los participantes. Se solicitó la autorización para la utilización del cuestionario a sus autores, al Ministerio de Salud de Colombia y a la representante para Colombia de la UNODC.

\section{Participantes}

Se incluyeron pacientes que asistían a los centros de atención de las drogodependencias de la Clínica Samein S.A.S y del Hospital San Vicente Fundación, los cuales atienden a afiliados del régimen contributivo y del subsidiado, así como a los servicios terapéuticos de la Corporación Cardynal y la Fundación Camino a la Vida, que atienden a usuarios particulares de diferentes municipios de Antioquia.

Los criterios de inclusión fueron los siguientes: ser de nacionalidad colombiana, tener más de 18 años, tener un consumo perjudicial de sustancias o un síndrome de dependencia a sustancias psicotrópicas, según los criterios de la Clasificación Internacional de Enfermedades (CIE-10), y estar recibiendo asistencia médica para abandonar el consumo de dichas sustancias.

Los criterios de exclusión fueron los siguientes: haber sido diagnosticado con retraso mental moderado o grave, o con demencia, ser analfabeta y tener exclusivamente consumo perjudicial o síndrome de dependencia al tabaco.

Se llevó a cabo un muestreo no probabilístico de manera secuencial incluyendo a los pacientes que cumplían los criterios de elegibilidad y estaban dispuestos a participar.

Se calcularon los respectivos tamaños de muestra para cada propiedad psicométrica. Para garantizar la consistencia interna, se calculó un tamaño de muestra de 98 participantes utilizando las fórmulas propuestas por Bonett (17), con un error de tipo I de 0,05, un valor esperado para el alfa de Cronbach de 0,7, y una amplitud del intervalo de confianza de 0,2, teniendo en cuenta que la subescala con el menor número de ítems tiene cuatro.

En cuanto a la confiabilidad entre evaluadores, se calculó un tamaño de muestra de 63 participantes utilizando la fórmula para medir la reproducibilidad (18) y asumiendo un error de tipo I de 0,05, uno de tipo II de 0,20, un coeficiente de correlación intraclase ( $\mathrm{CCl}$ ) especificado en la hipótesis nula de 0,5 y uno para la hipótesis alterna de 0,7 , y para dos evaluadores.

Para la confiabilidad prueba-reprueba, se calculó un tamaño de muestra de 41 participantes con un error de tipo I de 0,05 , un poder de 0,80 , un $\mathrm{CCl}$ de 0,7 y un nivel de precisión de 0,2, utilizando la fórmula basada en la amplitud del intervalo de confianza (19). 
Para la validez convergente se calculó un tamaño de muestra de 65 sujetos utilizando la fórmula de correlación (20) con un error de tipo I de 0,05 , un poder de 0,80 y una prueba de hipótesis a una cola en la que el coeficiente de correlación de Spearman para la población fue de 0,5 en la hipótesis alterna y de 0,1 en la hipótesis nula.

\section{Adaptación cultural}

Se utilizaron las versiones en el idioma original y la validada en España, las cuales fueron facilitadas por los grupos de la Universidad de Pennsylvania y la Universidad de Oviedo. Se siguieron los lineamientos de la International Test Commission (21) y se formó un comité de psiquiatras hablantes nativos del español, con conocimientos lingüísticos, y expertos en el tema de la validación de cuestionarios y en adicciones, quienes leyeron de forma independiente cada una de las preguntas de las versiones en inglés y español, señalando los términos que podían ser confusos para la población colombiana y proponiendo cambios. Asimismo, se anotaron los términos coloquiales para referirse a cada una de las sustancias que aparecen en la lista de alcohol y drogas del cuestionario.

Se discutió y se redactó cada una de las preguntas considerando entre los principales cambios la adopción de la sigla "ISA" para el cuestionario, dado que está ampliamente difundida, a pesar de que la traducción al español de la palabra severity sería 'gravedad'. También se ajustaron el nivel educativo (básica primaria, secundaria, media, técnica, profesional y posgrado) y se incluyó la Clasificación Internacional Uniforme de Ocupaciones adaptada para Colombia (CIUO) - 08 AC (22). Para los ajustes de la subescala "Legal" se contó con la asesoría de dos abogados, ya que las preguntas sobre delitos debían adaptarse al sistema penal colombiano.

La versión preliminar fue traducida al inglés por un traductor que desconocía el cuestionario original y esta versión en inglés se comparó con la original para detectar los ítems disímiles, los cuales fueron discutidos por el comité. No se encontraron cambios sustanciales en las frases, por lo que no fue necesaria una nueva traducción. Se redactó, entonces, una versión del cuestionario previa a la definitiva.

\section{Prueba piloto}

Esta se hizo en un grupo de 15 adultos con adicciones utilizando la versión previa del ISA-6. Se les solicitó que señalaran los ítems que no entendieran, que explicaran cómo entendían las preguntas en los ítems utilizados para calcular los puntajes y que describieran las dificultades que podría tener una persona para entender la escala. La mayoría de los participantes consideró que ninguno de los ítems era difícil de entender o irrelevante. El formato de la escala se ajustó con la señalización de los "saltos" de pregunta a pregunta.

Con estas adaptaciones, se obtuvo la versión final del ISA-6. Se elaboró el manual de uso, en el cual se describieron los procedimientos necesarios para la formulación de las preguntas y su calificación teniendo en cuenta dichas adaptaciones y el manual disponible en inglés.

\section{Procedimientos}

Un psiquiatra experto en el tema de adicciones, con certificación en la estrategia Treatnet, capacitó a los miembros del equipo de investigación y cada uno recibió el manual de uso para revisarlo sección por sección. 
Todos los sujetos que asistían a consulta o estaban internados en los centros participantes fueron evaluados por un psiquiatra para comprobar su elegibilidad y que hubieran recibido una explicación y hubieran firmado el consentimiento informado; después, se les hizo la entrevista con el ISA-6 en un consultorio disponible en la institución.

Las siguientes son las definiciones de los términos pertinentes empleados en el estudio.

Consistencia interna. Se entiende como el grado en el que se correlacionan los ítems que conforman una escala (23). Se estimó la consistencia interna de cada subescala, dado que se trataba de un cuestionario de múltiples dimensiones en el que las subescalas son independientes entre sí (24-26) y cada ítem se relaciona únicamente con la variable latente de la correspondiente subescala.

Confiabilidad entre evaluadores. Mide el grado de concordancia entre los puntajes de los pacientes evaluados por diferentes personas (23). Por ello, un subgrupo de los participantes fue evaluado por dos psiquiatras entrenados: uno de ellos dirigía la entrevista y el otro calificaba cada uno de los ítems de forma independiente, roles que intercambiaban a lo largo del estudio. Se decidió hacerlo de forma simultánea porque en los artículos consultados se ha reportado que la entrevista dura aproximadamente una hora (27), lo que podría afectar la participación de los individuos evaluados.

Confiabilidad prueba-reprueba. Mide el grado de correlación de los puntajes obtenidos en el cuestionario en dos momentos diferentes de tiempo, sin que se haya presentado variación en el constructo, en este caso, la gravedad de la adicción (23). Para ello, el mismo investigador que había hecho la primera entrevista hizo de nuevo aquellas preguntas de los ítems del ISA-6 que otorgan puntaje en el curso de los siete días posteriores a la primera entrevista, dado que se consideró que no se observarían cambios significativos en el estado clínico de las personas en lo tocante a las adicciones, y que la probabilidad de que el paciente y el entrevistador recordaran las respuestas sería menor.

Validez de constructo. Es el grado en el cual el cuestionario mide el constructo pertinente (23). Dada la ausencia de un patrón de referencia para la gravedad de la adicción, las hipótesis se probaron con respecto a dos aspectos. Primero, el convergente, en el que se asumió que los puntajes del ISA-6 se correlacionaban con los de otros cuestionarios que miden constructos similares (28) y que fueron empleados en una primera entrevista con un subgrupo de participantes. Se esperaba encontrar relación entre las subescalas de "Salud física" del ISA-6 y el resumen del componente físico (Physical Summary Component, PSC) del Cuestionario de Salud de 12 ítems (Short Form 12 Health Survey, SF-12); entre la "Psiquiátrica" y el resumen del componente mental (Mental Summary Component, MSC) de este mismo cuestionario; entre la subescala "Empleo" y el dominio "Trabajo" del Cuestionario para evaluación de la discapacidad de la Organización Mundial de la Salud (World Health Organization Disability Assessment Schedule, WHODAS 2.0); entre la subescala "Social - Problemas" y el dominio de "Relaciones" del WHODAS 2.0, así como entre las subescalas "Alcohol" y "Drogas" del ISA-6 y el Short Inventory of Problems (SIP), y, por último, entre la subescala "Legal" y los "Criterios diagnósticos de personalidad antisocial" del Manual diagnóstico y estadístico de los trastornos mentales (Diagnostic and Statistical Manual of Mental Disorders, DSM-5). 
En cuanto al aspecto discriminatorio o divergente, se evaluó si los puntajes del ISA-6 tenían una relación nula o baja con los puntajes de cuestionarios que evalúan un constructo diferente (28). En todas las subescalas se evaluó la correlación con el puntaje del dominio "Movilidad" del WHODAS 2.0, en el cual se miden las dificultades para actividades como ponerse de pie, moverse dentro de la casa y caminar distancias. Se escogió este dominio porque se ha comprobado que la adicción tiene poco efecto en él (29).

\section{Instrumentos de medición}

Addiction Severity Index (ISA-6). Es una escala diseñada por un grupo de investigadores de la Universidad de Pennsylvania de Estados Unidos, que se aplica mediante entrevista semiestructurada y evalúa las alteraciones en las áreas de la vida del individuo que resultan afectadas por la adicción.

En esta escala el concepto de gravedad equivale a la necesidad de tratamiento adicional. La sexta edición se publicó en el 2006 y ofrece la ventaja de reducir el tiempo de la entrevista, pues al inicio de cada área hay "saltos", o preguntas de tamización, que, en caso de ser negativas, permiten continuar con la siguiente (30). Asimismo, aporta información sobre problemas en dichas áreas en los treinta días anteriores a la entrevista y a lo largo de la vida, pero, a diferencia de las versiones anteriores, incluye un puntaje para los últimos seis meses, período que se considera apropiado para los análisis económicos (11).

En cada subescala se explora el efecto del consumo y se registran datos relevantes para la historia clínica de ingreso. Con algunas de las preguntas se calculan los puntajes. En la subescala de "Salud física" se puntúan la percepción de la salud física del paciente, los días que ha presentado algún problema de salud física, la preocupación que este genera y la necesidad de tratamiento. La subescala de "Empleo" se califica a partir de la situación laboral del paciente en el momento de la entrevista, el dinero devengado en el último mes, los días que ha trabajado con remuneración y aquellos en que ha presentado problemas en el trabajo. Las de "Alcohol" y "Drogas" son subescalas que evalúan el consumo en el mes anterior, los problemas derivados de dicho consumo, el dinero gastado y la preocupación por el tratamiento y la importancia que el paciente le da. La subescala "Legal" puntúa los días de participación en actividades ilegales. La "Social" califica la relación con niños ("Social - Niños"), la percepción de apoyo por parte de la pareja, la familia y los amigos ("Social - Apoyo") y la presencia de problemas en las relaciones ("Social-Problemas"). En la subescala "Estado psiquiátrico" se determinan los días en que el sujeto ha presentado problemas psicológicos y la imposibilidad subsiguiente de realizar actividades, así como la molestia que generan y la importancia que otorga el paciente a su tratamiento. Las respuestas de naturaleza cuantitativa con nivel de medición de razón se convierten a un nivel de intervalo, según las indicaciones del grupo que desarrolló el cuestionario.

Con esta información, se calcula un puntaje crudo para cada subescala que luego se transforma en un puntaje T, siguiendo, también en este caso, los lineamientos de los autores de la escala (11). Al final, el evaluador califica la confiabilidad del entrevistador: pobre, si la información es inexacta o contradictoria; regular, si el perfil parece razonable, aunque con numerosas inexactitudes aparentes en una o dos áreas, y buena, si el perfil parece ser un buen reflejo del entrevistado o hay pocas inexactitudes. La sexta versión solo ha sido validada en Brasil (12). 
Cuestionario de Salud de 12 ítems (SF-12). Evalúa de manera genérica la calidad de vida relacionada con la salud y permite calcular dos medidas de resumen del estado de salud: el CSC y el MSC. Dichas medidas indican la funcionalidad física y mental del individuo y los puntajes bajos indican una menor calidad de vida. De ahí que se espere una correlación negativa moderada con los puntajes de gravedad de las subescalas de "Salud física" y "Psiquiátrica" del ISA-6. Se escogió porque el marco de referencia temporal es el momento actual (últimas cuatro semanas) y proporciona una medida de la salud física y mental percibida por el mismo sujeto con adicción. También se ha demostrado su concordancia con el SF-36 en población adicta en terapia de suplencia con metadona (31) y se ha empleado en validaciones del ISA-6 $(11,12)$. Esta escala se validó en Colombia y se ha comprobado que tiene adecuadas propiedades psicométricas $(32,33)$.

Cuestionario para la evaluación de la discapacidad de la Organización Mundial de la Salud (WHODAS 2.0). Es un instrumento genérico para la evaluación de la salud y la discapacidad (34). Evalúa la capacidad del paciente para desempeñar actividades en seis dominios diferentes en los 30 días anteriores. Se ha comprobado que tiene buenas propiedades psicométricas en pacientes con trastornos psiquiátricos $(35,36)$ y se lo ha propuesto para calificar su funcionalidad $(37,38)$. En pacientes con adicciones se ha encontrado una mayor discapacidad en todos los dominios en comparación con la población general (39), aunque el menos afectado es el de la movilidad (29). Se utilizó el dominio de "Relaciones interpersonales" y se esperaba una correlación positiva moderada con la subescala "Social Problemas" del ISA-6, así como una correlación positiva moderada entre el dominio "Trabajo" y la subescala "Empleo"; por último, se utilizó el dominio de "Movilidad", con el que se esperaban correlaciones nulas o bajas con todas las subescalas del ISA-6.

Short Inventory of Problems (SIP). Es un cuestionario que diligencia la propia persona y que inquiere sobre la frecuencia de diversas consecuencias del uso del alcohol y las drogas en el mes anterior. Los puntajes altos reflejan una mayor frecuencia de los problemas relacionados con el consumo. En los estudios de validación de su versión original en inglés y de la traducción al español, se ha encontrado que su confiabilidad y validez son buenas (40-42). Este instrumento se ha utilizado para la validación de varias versiones del ISA $(25,26)$. En este estudio se esperaban correlaciones positivas moderadas entre el puntaje del SIP y el puntaje de las subescalas de "Alcohol" y "Drogas" del ISA-6.

Criterios diagnósticos de personalidad del DSM-5. Se evaluaron los criterios diagnósticos del trastorno de personalidad antisocial del DSM5 , específicamente los siete indicadores del criterio $A(A 1-A 7)$, que corresponden a situaciones y patrones de conducta que sugieren la violación y la despreocupación por los derechos de los demás y que, con frecuencia, llevan a la formulación de cargos en procesos legales (37). Se esperaba una correlación positiva moderada entre el número de indicadores del criterio $A$ y el puntaje de la subescala "Legal" del ISA-6.

\section{Análisis estadístico}

Las características sociodemográficas y clínicas de los participantes se resumieron mediante procedimientos de estadística descriptiva: frecuencias y porcentajes para las variables categóricas, y medianas y rangos intercuartílicos para las variables continuas (dado que no seguían una distribución normal según la prueba de Shapiro-Wilk con valores de 
$p<0,05$ para todas). Asimismo, se presentaron medidas de tendencia central y de posición de las puntuaciones en cada subescala. Se evaluó si había un efecto piso o un efecto techo cuando más del $15 \%$ de los pacientes alcanzaban la mínima o la máxima puntuación posible, respectivamente (43).

En cuanto a la consistencia interna, se calculó el alfa de Cronbach y se le consideró adecuado si los valores fluctuaban entre 0,7 y 0,9 (44). Para la confiabilidad entre evaluadores y la de prueba-reprueba, se calculó el CCI del tipo de acuerdo absoluto entre las puntuaciones de cada subescala del ISA-6 en las dos mediciones, con sus respectivos intervalos de confianza del $95 \%$ $\left(\mathrm{IC}_{95 \%}\right)$, y se consideró como apropiada una correlación con valores mayores de 0,7 (43). Asimismo, se hizo el gráfico de Bland y Altman y se establecieron los límites de acuerdo para la confiabilidad prueba-reprueba.

Para evaluar la validez convergente, se calculó el coeficiente de correlación de Spearman ( $r$ ), con sus respectivos valores de $p$; se esperaba que este arrojara valores por encima de 0,5 , lo que indica correlaciones moderadas, en tanto que para la validez divergente se esperaba un valor de $r$ menor de 0,3, es decir, una correlación baja (45). En todos los análisis se utilizó el programa estadístico para ciencias sociales IBM SPSS ${ }^{\mathrm{TM}}$, versión 23.

\section{Resultados}

\section{Características de la muestra}

Se incluyeron 153 sujetos con adicciones, la mayoría de ellos hombres en la cuarta década de la vida, solteros, y una mediana de escolaridad de 11 años (cuadro 1). El 91,59 \% de la muestra pertenecía al régimen contributivo del sistema de salud. Entre las mujeres había una embarazada.

En cuanto a la situación laboral, más de la mitad de los sujetos incluidos no estaba trabajando en el momento de la evaluación; entre quienes trabajaban, el $70,4 \%$ no refirió haber tenido problemas relacionados con el trabajo en ninguno de los días.

En la esfera legal, el 42,2\% de los participantes admitió haber sido arrestado alguna vez en la vida, y el 16,3\% refirió haber participado en actividades ilegales durante el mes anterior.

En cuanto a la situación social, cerca de un tercio de los pacientes convivía con menores de 18 años, y de ellos, el 9,15\% percibía algún grado de dificultad en la relación con los niños. El 63,4 \% reportó haber tenido problemas en la relación con la pareja, la familia o los amigos y en el 18,3 $\%$ de los casos, las interacciones con ellos habían terminado en golpes 0 tirando objetos.

La decisión de iniciar el tratamiento había recaído en el paciente, la familia o los amigos en el $85 \%$ de los casos. Se incluyeron pacientes con adicción exclusivamente al alcohol (15\%), exclusivamente a drogas (28,8 $\%)$ y a ambos (56,3\%). En el cuadro 1 se presentan otras características adicionales de los sujetos de la muestra.

\section{Características de la escala}

La entrevista con el ISA-6 duró, en promedio, 46,76 minutos. En el cuadro 2 se presenta el resumen de los puntajes crudos y los trasformados en puntajes T en cada subescala. Se observó un efecto piso en las subescalas 
"Legal" y "Social - Niños", pues el 83,7 \% de los participantes obtuvo un puntaje crudo de cero en la primera y el $73,9 \%$ en la segunda. No se encontró un efecto techo en esta muestra. En los ítems que permiten el cálculo de los puntajes de cada subescala no se presentaron pérdidas, por lo que no fue necesaria la imputación de datos.

Cuadro 1. Características sociodemográficas y clínicas de 153 sujetos con adicciones incluidos en la validación del ISA-6 en Colombia

\begin{tabular}{|c|c|c|}
\hline Características sociodemográficas y clínicas & Mediana & Rango intercuartílico \\
\hline Edad (años) & 33 & 24 a 44 \\
\hline Escolaridad (años) & 11 & 11 a 13 \\
\hline Hospitalizaciones por enfermedad física & 1 & 0 a 3 \\
\hline Días con problemas de salud física en el último mes & 3 & 0 a 8 \\
\hline Hospitalizaciones por trastorno psiquiátricos & 0 & 0 a 1 \\
\hline Días con problemas psicológicos en el último mes & 5 & 0 a 19 \\
\hline Años de consumo regular de alcohol & 5 & 0 a 17,5 \\
\hline Días de uso de alcohol en el último mes & 4 & 2 a 11 \\
\hline Años de consumo regular de drogas & 11 & 7 a 18,5 \\
\hline Días de uso de drogas en el último mes & 15 & 5 a 28 \\
\hline \multirow[t]{2}{*}{ Número de tratamientos para adicciones } & 2 & 1 a 3 \\
\hline & Frecuencia & Porcentaje \\
\hline \multicolumn{3}{|l|}{ Sexo } \\
\hline Masculino & 136 & 88,9 \\
\hline \multicolumn{3}{|l|}{ Estado civil } \\
\hline Soltero & 87 & 56,9 \\
\hline Unión libre/casado & 40 & 26,1 \\
\hline Separado/divorciado & 24 & 15,7 \\
\hline Viudo & 2 & 1,3 \\
\hline \multicolumn{3}{|l|}{ Situación laboral } \\
\hline Empleado & 71 & 46,4 \\
\hline \multicolumn{3}{|l|}{ Salud física } \\
\hline Antecedente de comorbilidad médica & 56 & 36,6 \\
\hline \multicolumn{3}{|l|}{ Esfera legal } \\
\hline Relata arresto alguna vez en la vida & 63 & 41,2 \\
\hline \multicolumn{3}{|l|}{ Situación social } \\
\hline Ha vivido con niños en el último mes & 53 & 34,6 \\
\hline Percibe ayuda en su red social & 152 & 99,4 \\
\hline Reporta problemas de relación & 97 & 63,4 \\
\hline \multicolumn{3}{|l|}{ Modalidad de tratamiento de adicciones } \\
\hline Residencial & 47 & 30,7 \\
\hline Ambulatorio & 67 & 43,8 \\
\hline Consulta externa & 39 & 25,5 \\
\hline \multicolumn{3}{|l|}{ Sustancia principal } \\
\hline Alcohol & 39 & 25,5 \\
\hline Marihuana & 37 & 24,2 \\
\hline Cocaína & 67 & 43,8 \\
\hline Heroína & 5 & 3,3 \\
\hline Otras & 5 & 3,3 \\
\hline \multicolumn{3}{|l|}{ Fiabilidad } \\
\hline Buena & 111 & 72,5 \\
\hline Regular & 35 & 22,9 \\
\hline Pobre & 7 & 4,6 \\
\hline
\end{tabular}


Cuadro 2. Puntajes crudos y transformados en puntajes $T$ de 153 sujetos con adicciones en Colombia entrevistados con el ISA-6

\begin{tabular}{lcccc}
\hline \multirow{2}{*}{ Subescala } & \multicolumn{2}{c}{ Puntaje crudo } & \multicolumn{1}{c}{ Puntaje T } \\
\cline { 2 - 5 } & Mediana & Rango ontercuartílico & Mediana & Rango intercuartílico \\
\hline Salud física & 8,0 & 3,0 a 12,0 & 45 & 39 a 50 \\
Empleo & 10,8 & 1,6 a 14,6 & 43 & 28 a 53 \\
Alcohol & 6,0 & 3,0 a 15,3 & 50 & 15 a 29 \\
Drogas & 15,0 & 4,0 a 29,4 & 47 & 36 a 53 \\
Legal & 0,0 & 0,0 a 0,0 & 46 & 46 a 46 \\
Social - Niños & 0,0 & 0,0 a 1,0 & 48 & 48 a 56 \\
Social - Apoyo & 14,0 & 11,0 a 15,0 & 69 & 58 a 69 \\
Social - Problemas & 7,0 & 4,0 a 10,0 & 53 & 46 a 60 \\
Psiquiátrica & 15,9 & 8,9 a 23,9 & 46 & 42 a 51 \\
\hline
\end{tabular}

\section{Consistencia interna}

Se había propuesto un tamaño muestral de 98 sujetos para esta propiedad, pero finalmente se evaluaron 153, pues fue posible hacer la entrevista a un mayor número de participantes. En la mayoría de las subescalas del ISA-6 se encontró una consistencia interna adecuada, con un alfa de Cronbach por encima de 0,7. En ninguna de las subescalas se encontraron valores que sugirieran ítems redundantes. En el cuadro 3 se presentan los valores de este estadístico para cada subescala. En la "Social - Problemas", el alfa de Cronbach obtenido fue ligeramente menor de 0,7.

Cuadro 3. Consistencia interna y confiabilidad del ISA-6 en sujetos con adicciones en Colombia

\begin{tabular}{lcccccc}
\hline \multirow{2}{*}{ Subescala } & $\begin{array}{c}\text { Número de } \\
\text { ítems }\end{array}$ & $\begin{array}{c}\text { Consistencia interna } \\
(\mathbf{n = 1 5 3 )}\end{array}$ & \multicolumn{2}{c}{$\begin{array}{c}\text { Confiabilidad entre } \\
\text { evaluadores (n=64) }\end{array}$} & \multicolumn{2}{c}{$\begin{array}{c}\text { Confiabilidad } \\
\text { prueba-reprueba (n=45) }\end{array}$} \\
\cline { 3 - 7 } & & Alfa de Cronbach & $\mathbf{C C l}$ & $\mathbf{I C}_{\mathbf{9 5}} \%$ & $\mathbf{C C l}^{\mathbf{C}}$ & $\mathbf{I C}_{\mathbf{9 5}} \%$ \\
\hline Salud física & 7 & 0,83 & 0,92 & $0,87-0,95$ & 0,71 & $0,52-0,83$ \\
Empleo & 4 & 0,89 & 0,98 & $0,97-0,99$ & 0,98 & $0,97-0,99$ \\
Alcohol & 11 & 0,73 & 0,83 & $0,74-0,89$ & 0,78 & $0,63-0,87$ \\
Drogas & 12 & 0,90 & 0,93 & $0,88-0,95$ & 0,75 & $0,58-0,86$ \\
Legal & 6 & 0,74 & 0,97 & $0,95-0,98$ & 0,97 & $0,95-0,98$ \\
Social - Niños & 5 & 0,70 & 0,98 & $0,97-0,99$ & 0,84 & $0,72-0,91$ \\
Social - Apoyo & 4 & 0,76 & 0,91 & $0,85-0,94$ & 0,84 & $0,74-0,91$ \\
Social - Problemas & 5 & 0,66 & 0,98 & $0,97-0,99$ & 0,72 & $0,54-0,83$ \\
Psiquiátrica & 16 & 0,81 & 0,95 & $0,93-0,96$ & 0,85 & $0,73-0,91$ \\
\hline
\end{tabular}

CCI: coeficiente de correlación intraclase; IC95\%: intervalo de confianza del $95 \%$

\section{Confiabilidad entre evaluadores}

Dado que los evaluadores pueden ser fuente de variabilidad, se evaluaron 64 sujetos, uno más de los contemplados inicialmente. Se encontró que los puntajes de todas las subescalas del ISA- 6 reflejaban una gran confiabilidad (cuadro 3). El menor CCI se calculó para la subescala "Alcohol", con 0,83, lo que indicaba que el $83 \%$ de la variabilidad total de la puntuación se explicaba por la verdadera variabilidad entre los pacientes con adicción al consumo de alcohol, en tanto que la restante se explicaría por las diferencias entre los dos evaluadores, y por el azar, lo cual se considera adecuado.

\section{Confiabilidad prueba-reprueba}

Aunque se planeó evaluarla en 41 participantes, las dos evaluaciones pudieron hacerse en 45 sujetos. Los puntajes obtenidos en dichas evaluaciones reflejaban una confiabilidad alta para todas las subescalas 

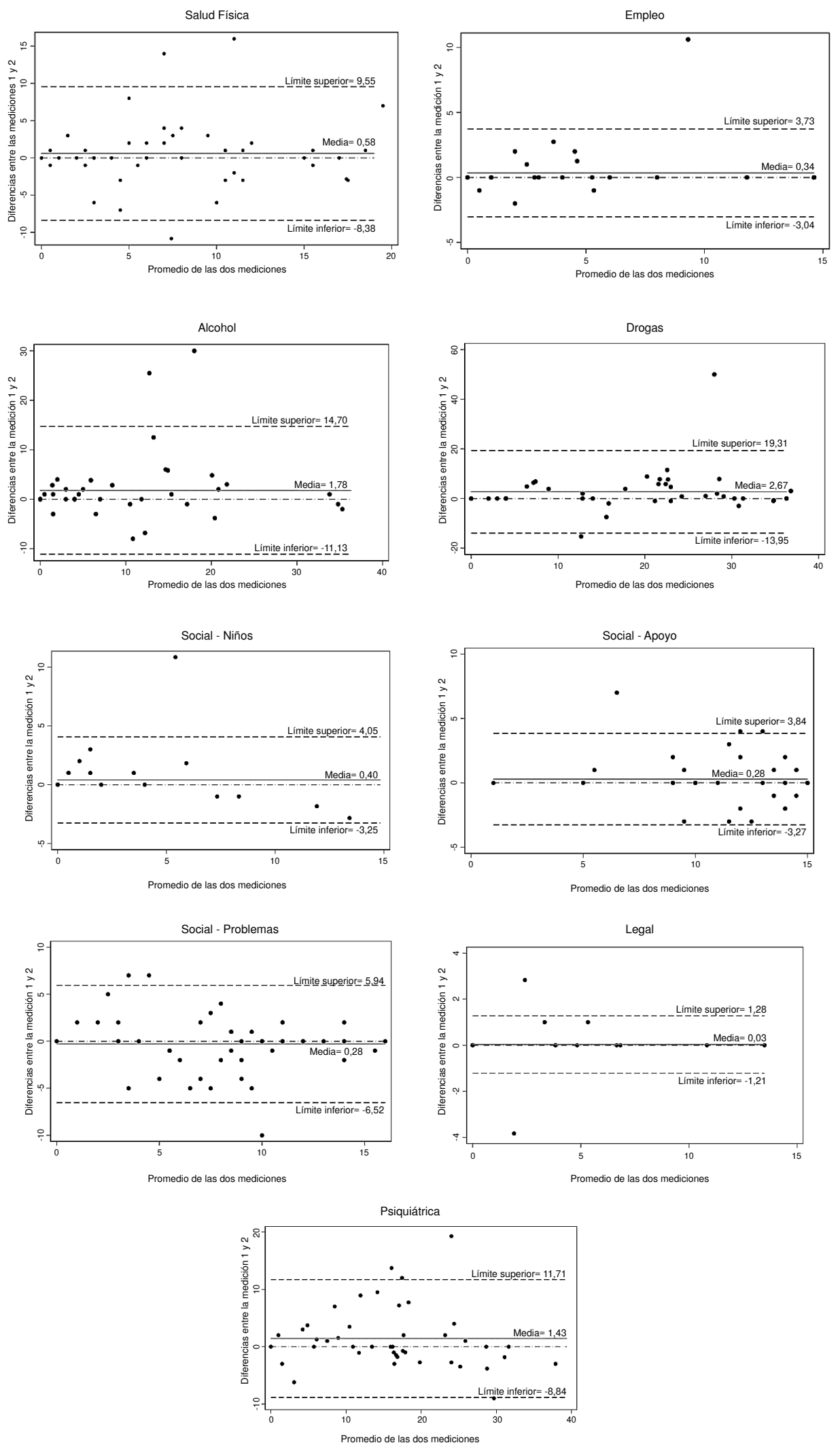

Figura 1. Confiabilidad prueba-reprueba del ISA-6. Se muestra el gráfico de Bland y Altman para cada subescala. Las líneas punteadas representan los límites del acuerdo de $95 \%$ (superior e inferior) y la línea continua, la media. 
del ISA-6 (cuadro 3), con un CCI que osciló entre 0,71 y 0,98 (subescalas de "Salud física" y "Empleo", respectivamente). Mediante el gráfico de Bland y Altman (figura 1) fue posible confirmar que el acuerdo entre las dos mediciones era alto y que las diferencias observadas no siguieron un patrón determinado, es decir, podrían deberse más al azar.

\section{Validez de constructo}

Aunque inicialmente se calculó una muestra de 64 individuos, fue posible aplicar el ISA-6 y los demás instrumentos a 84 sujetos. Se encontró que cada una de las subescalas del ISA-6 fue válida en cuanto al aspecto convergente del constructo, con correlaciones altas y moderadas indicadas por un valor de ro mayor de 0,5 (cuadro 4).

Cuadro 4. Validez de constructo del ISA-6 comparado con otros cuestionarios en 153 sujetos con adicciones en Colombia

\begin{tabular}{lccccccc}
\hline \multicolumn{1}{c}{ Subescala } & $\begin{array}{c}\text { SF-12 } \\
\text { PSC }\end{array}$ & $\begin{array}{c}\text { WHODAS } \\
\text { Empleo }\end{array}$ & SIP & Anti-social & $\begin{array}{c}\text { WHODAS } \\
\text { Relación }\end{array}$ & $\begin{array}{c}\text { SF-12 } \\
\text { MSC }\end{array}$ & $\begin{array}{c}\text { WHODAS } \\
\text { Movilidad }\end{array}$ \\
\hline Salud física & $-0,72^{*}$ & - & - & - & - & - & $0,34^{*}$ \\
Empleo & - & $0,88^{*}$ & - & - & - & - & 0,10 \\
Alcohol & - & - & $0,79^{*}$ & - & - & - & 0,29 \\
Drogas & - & - & $0,74^{*}$ & - & - & - & $-0,12$ \\
Legal & - & - & - & $0,57^{*}$ & - & - & 0,10 \\
Social-Problema & - & - & - & - & $0,53^{*}$ & - & 0,15 \\
Psiquiátrico & - & - & - & - & - & $-0,85^{*}$ & 0,14 \\
\hline
\end{tabular}

Los valores corresponden al coeficiente de correlación de Spearman (ro).

* Valor de $\mathrm{p}<0,01$

SF-12: Short Form 12 Health Survey. PSC: resumen del componente físico. MSC: resumen del componente mental. WHODAS: Cuestionario para la evaluación de discapacidad de la Organización Mundial de la Salud. SIP: Short Inventory of Problems

En la subescala de "Salud física" se encontró una elevada correlación negativa con el PSC del SF-12, lo que indica que a mayor gravedad de la adicción, menor la funcionalidad en el componente físico de la calidad de vida.

La subescala "Psiquiátrica" presentó una alta correlación negativa con el MSC del SF-12, lo que sugiere que, tras aumentar la gravedad de los problemas psiquiátricos, disminuyó la percepción de la calidad de vida en cuanto a la funcionalidad mental.

La gravedad en la subescala de "Empleo" medida con el ISA-6 tuvo una elevada correlación positiva con la discapacidad laboral medida mediante el WHODAS 2.0.

Los puntajes de las subescalas de "Alcohol" y "Drogas" tuvieron una elevada correlación positiva con el SIP.

La subescala "Legal" tuvo una correlación positiva moderada con los criterios diagnósticos del trastorno de personalidad antisocial del DSM-5, al igual que la subescala "Social - Problemas" con la discapacidad en las relaciones medida por el WHODAS 2.0.

En cuanto al aspecto divergente, no se encontró correlación entre las subescalas del ISA-6 y la discapacidad generada por la movilidad medida con el WHODAS 2.0, salvo en la subescala de "Salud física", con una baja correlación positiva ( $\mathrm{ro}=0,34)$, lo cual sugeriría que al aumentar los problemas físicos, se produciría un discreto aumento en la discapacidad para la ejecución de movimientos y desplazamientos. 


\section{Discusión}

Se hizo la adaptación cultural del ISA-6 y se encontró que presentaba una consistencia interna, una confiabilidad entre evaluadores y de pruebareprueba, así como una validez de constructo, adecuadas.

La mayoría de los participantes eran hombres bachilleres, principalmente entre los 22 y 44 años, y desempleados. Una característica que se evidenció en las medidas de posición (cuartiles) fue la heterogeneidad en las variables medidas en casi todas las subescalas, lo cual indica que se incluyeron sujetos con adicciones de diferente gravedad. Estos rasgos coinciden con los reportes de las características de los pacientes que consultan los centros de atención de las drogodependencias en otros lugares de Colombia (46) y en los sujetos incluidos en los procesos de derivación y validación de la misma versión de la escala $(11,12)$.

Esto resulta ventajoso en la medida en que facilita la comparación de los resultados y permite, según la teoría clásica de los tests, apreciar mejor la confiabilidad de un cuestionario porque se incluyen individuos que responden en diferente grado al constructo (43). No obstante, se observó un efecto piso en las subescalas "Legal" y "Social - Niños". Podría sugerirse la inclusión de otros ítems en los puntajes para discriminar mejor esta población, pues es probable que este resultado se haya debido a la distribución del rasgo en la muestra, ya que no se incluyeron muchos sujetos que hubieran cometido delitos ni que hubieran convivido con niños en el mes anterior. Esto debe tenerse en cuenta en futuros análisis longitudinales de los puntajes de estas subescalas, pues es probable que, en caso de que los individuos presentaran alguna mejoría, esta no fuera detectable.

En cuanto a la consistencia interna, al igual que en los otros estudios de validación de la misma versión del ISA-6, se encontró que fue adecuada en las subescalas del ISA-6 y fue baja solo en la subescala "Social Problemas", lo cual también sucedió en la validación llevada a cabo en Brasil, en la que el alfa de Cronbach para esta subescala fue de 0,64 , el más bajo (12). En la validación en España, el grado de consistencia interna estuvo entre 0,85 y 0,95, excepto para las subescalas "Legal" $(0,47)$, "Social-Niños" $(0,58)$ y "Social-Problemas" $(0,70)(13)$.

Una posible explicación de dicho resultado en esta última subescala sería la divergencia entre la presencia de problemas y discusiones con la pareja, los familiares y amigos, que son los ítems que la conforman, en contraste con la preocupación y la importancia del tratamiento para el paciente. Puede ser que el relato de las dificultades no esté ligado a la percepción del tratamiento, o no genere una gran preocupación en los pacientes. En general, los jóvenes tienden a infravalorar el problema de las adicciones y eso influye en su apreciación de los sucesos problemáticos y conlleva una menor preocupación (47).

En los modelos de relaciones sociales con los que se analizan y cuantifican los elementos de la interacción interpersonal, se ha encontrado que la percepción es una fuente de varianza (es decir, lo que relata el sujeto con adicción), lo que difiere de las conductas y del objetivo (48) que, en este caso, serían la familia, la pareja o los amigos, a quienes no se entrevistó directamente. De esta forma, se estarían incluyendo en la misma subescala dos aspectos conceptuales que no pueden escindirse y que, a pesar de la inestabilidad en la consistencia, se deben mantener. 
También se encontró una elevada confiabilidad de los evaluadores en el empleo de la versión adaptada del ISA-6, con un $\mathrm{CCI}$ por encima de 0,83 para todas las subescalas. Estos valores fueron superiores a los reportados por Kessler, et al. (12), quienes evaluaron separadamente a 41 pacientes y compararon los puntajes obtenidos por dos evaluadores, sin aclarar el tiempo de diferencia entre una y otra entrevista. Los autores encontraron una correlación moderada para las subescalas de "Salud física" $(\mathrm{CCl}=0,55$; $\left.\mathrm{IC}_{95 \%}: 0,29-0,73\right)$ y la "Psiquiátrica" $\left(\mathrm{CCl}=0,67 ; \mathrm{IC}_{95 \%}: 0,45-0,81\right)$ y alta para las demás subescalas (CCl mayor de 0,7$)$. Es probable que se sobreestime la confiabilidad de las mediciones de dos evaluadores cuando se califica de forma simultánea en una misma entrevista.

La confiabilidad prueba-reprueba indica la estabilidad de los puntajes de la prueba en un intervalo de tiempo en el que no se han presentado cambios en el constructo. En este estudio se encontró una correlación alta entre los puntajes obtenidos en la evaluación inicial y en la repetida en el curso de la semana siguiente por el mismo evaluador, con un $\mathrm{CCl}$ superior a 0,72. Para evaluar dicha confiabilidad, en Brasil se utilizó la versión adaptada en el momento del ingreso al tratamiento de la adicción y a 51 de los sujetos se les repitió en el curso de los tres a siete días posteriores a cargo de un calificador diferente. A pesar de la potencial fuente de variación que representaría un entrevistador diferente, los autores encontraron un $\mathrm{CCl}$ mayor de 0,73 para todas las subescalas (12). En España se administró la escala en dos ocasiones diferentes separadas por una media de tiempo de 21,8 días (desviación estándar, $\mathrm{DE}=12,6)$. Las autores reportaron un valor de ro que osciló entre 0,36 ("Social - Problemas") y 0,96 ("Social - Apoyo") y 1 ("Social Hijos") (13). Es probable que la baja confiabilidad observada en algunas subescalas se deba al amplio intervalo de tiempo, durante el cual podrían haberse dado cambios en el constructo, aspecto reflejado por la escala de forma confiable.

Se confirmaron también las hipótesis sobre la relación del ISA-6 con otros cuestionarios que se aproximan a la dimensión medida por cada subescala. El consumo de tóxicos tiene diversas implicaciones para la salud física, esto por el efecto derivado de la sustancia, la vía de administración, las conductas de riesgo asociadas, y los síntomas físicos de la abstinencia, entre otros (49-51).

En el SF-36 se ha visto que la percepción de salud de los pacientes está relacionada con la evaluación de la gravedad de su adicción por parte del clínico (52), por lo que la puntúan con menores valores en comparación con la población general (53).

En este estudio se encontró una correlación de $-0,72$ con el PSC de la SF12, lo que indica que cuanto mayor es el deterioro físico, menor es la calidad de vida. En el proceso de remisión de pacientes con base en los resultados del ISA-6 en Estados Unidos, se evaluó esta misma relación y se encontró una correlación de -0,64 (11). En Brasil esta subescala se comparó con el dominio físico de la escala de calidad de vida de la Organización Mundial de la Salud (World Health Organization Quality of Life, WHOQOL-BREF) y se calculó un coeficiente de correlación de Pearson $(r)$ de -0,41 $(p<0,05)(12)$.

La salud mental también resulta afectada con las adicciones. Se han encontrado con mayor frecuencia síntomas ansiosos, psicóticos y depresivos que incluyen la conducta suicida, así como alteraciones en la conducta y en los ritmos biológicos, en pacientes con adicción al consumo de alcohol y de drogas (54-57), síntomas estos que se relacionan con una peor calidad de vida (58). En esta validación, la correlación entre la gravedad de la subescala 
"Psiquiátrica" fue negativa y alta, con un MSC del SF-12 $(r=-0,85)$ similar a lo encontrado en la remisión basada en el cuestionario $(-0,61)$. Con la versión brasileña se encontró una alta correlación indirecta con el dominio psicológico de la WHOQOL-BREF $(r=-0,75)$. Todo esto indica que el efecto de la adicción en la salud física y mental puede evaluarse de forma válida mediante el ISA-6.

El efecto de la adicción en la situación laboral también es evidente. La incidencia del consumo es mayor entre los individuos que están desempleados, y los individuos con adicción pierden con frecuencia sus trabajos, lo que se ha relacionado con más recaídas y un aumento en el patrón de consumo $(59,60)$.

Con el WHODAS 2.0 se evaluó la discapacidad que experimentaban los pacientes en el trabajo y se encontró una correlación alta con la gravedad de la adicción en la subescala "Empleo". Esta misma subescala se comparó en la remisión, y en la validación en Brasil del ISA-6, con el desempeño laboral medido con la Social Adjustment Scale Self-Report (SAS-SR) y se encontró una correlación positiva $(r=0,76$ y 0,47 , respectivamente) $(11,12)$.

Las relaciones sociales también se ven afectadas por el consumo de tóxicos. Las discusiones, las amenazas, el maltrato y la violencia contra la pareja o la familia son frecuentes, así como el aumento en los gastos familiares y la negligencia en los deberes (61-63). Con el WHODAS 2.0 se evaluaron las dificultades en las interacciones con los demás (pareja, familia o amigos) y se encontró una correlación positiva significativa con la subescala "Social - Problemas", en la cual, precisamente, se incluyen este tipo de conflictos.

En los estudios en los que se han contrastado los puntajes del área social de la SAS-SR y el ISA-6 $(11,12)$, los coeficientes de correlación han sido bajos $(0,4$ y 0,25$)$. Es probable que ello se explique por la diferencia de constructos, pues en la SAS-SR se evalúa el desempeño en las últimas dos semanas en las relaciones sociales, específicamente en el desempeño en las tareas esperadas, las asperezas con los miembros de la familia, los sentimientos y las satisfacciones (64). La subescala "Social - Problemas", en cambio, puntúa los problemas de relación y las discusiones con la pareja, la familia y los amigos, el desenlace violento de las interacciones, el grado de preocupación por estos problemas y la necesidad de una asesoría en este sentido. Es probable que el puntaje de discapacidad social del WHODAS 2.0 ofrezca una visión cercana al de las consecuencias sociales del consumo.

La estimación de la validez de constructo de la subescala "Legal" es compleja dada la ausencia de un instrumento que evalúe directamente la comisión de delitos en el mes anterior. En este estudio se comparó el puntaje de esta dimensión con el número de indicadores del criterio A para el diagnóstico del trastorno de personalidad antisocial, y se obtuvo una correlación significativa. Por el contrario, en Estado Unidos la hipótesis de validez de constructo se probó con el número de arrestos en los dos años anteriores y la correlación fue baja $(r=0,15)$; también se comparó con la escala de socialización del California Psychological Inventory, en la que se puntúan las fortalezas del paciente para socializar y aceptar reglas sociales según su propio reporte, y se encontró una correlación negativa baja $(r=-0,14)(11)$. Una posible explicación es que no todos los actos ilegales conducen necesariamente a arrestos; también es posible que las escalas diligenciadas por los propios pacientes no sean indicadores reales de rasgos antisociales, precisamente por el sesgo que implican características nucleares antisociales como la mentira y la manipulación (65). 
En Brasil se comparó el comportamiento de esta subescala con el dominio de "Ambiente" de la WHOQOL-BREF, el cual incluye las fuentes financieras y la seguridad, pero era de esperarse una correlación baja $(r=0,42)$, dado que son constructos diferentes (12).

Para las subescalas de "Alcohol" y "Drogas", la validez de constructo se evalúo comparando con el SIP, y se obtuvo una correlación alta. En el estudio de la interconsulta empleando el ISA-6, se encontró una relación similar $(r=0,69$ para "Alcohol" y r=0,61 para "Drogas") (11). En Brasil se encontró una relación ( $r=0,72$ para "Alcohol" y $r=0,89$ para "Drogas") con el Alcohol, Smoking and Substance Involvement Screening Test (ASSIST) desarrollado para detectar el abuso y la dependencia de sustancias (12). La validez de constructo en España se midió para todas las subescalas con la escala de Clinical Global Impression (CGl) sin ninguna modificación y todas las correlaciones estuvieron por debajo de 0,24 (13), lo cual era previsible, pues es una escala que informa de manera general qué tan enfermo se encuentra el paciente (66).

En cuanto al aspecto divergente, se encontró únicamente una correlación entre la subescala de "Salud física" y la discapacidad en la movilidad del WHODAS 2.0. Es probable que entre los síntomas físicos calificados con el ISA-6, se incluyeran el dolor de origen musculo-esquelético con efecto en la movilidad y la capacidad para ponerse de pie, los cuales serían dos constructos relacionados conceptualmente.

Es posible ver, entonces, que en pacientes en quienes no ha cambiado la gravedad de la adicción, el grado de los puntajes de las diferentes subescalas del ISA-6 son los mismos cuando diferentes evaluadores evalúan diferentes grupos de ítems en un corto periodo. Asimismo, la gravedad de la adicción y sus consecuencias físicas, mentales, laborales, sociales y legales quedan adecuadamente reflejadas en el cuestionario y, por ello, este puede utilizarse en el medio colombiano para evaluar pacientes con adicciones que buscan tratamiento.

Entre las limitaciones de este estudio se debe mencionar la inclusión de una mayoría de individuos con un alto nivel educativo y perteneciente al régimen contributivo del sistema de salud, lo que puede afectar la generalización de los datos, pues algunas de las preguntas del instrumento requerirían habilidades matemáticas y una apreciable capacidad de abstracción.

Por ello es probable que sean necesarios estudios adicionales que incluyan pacientes con otras características. Asimismo, la confiabilidad de los evaluadores pudo haberse sobreestimado, ya que el ISA-6 es una entrevista semiestructurada y pueden darse variaciones en la formulación de las preguntas por parte de cada entrevistador $y$, por ende, generarse respuestas diferentes que afecten la puntuación. También existe un sesgo inherente a la población de estudio y es la deseabilidad social.

En general, la conducta adictiva lleva inherente una carga social, y es posible que los participantes tiendan a minimizar la información o la gravedad de su situación para evitar ser juzgados o complacer a los terapeutas. Aunque no era parte del objetivo de este estudio, es importante mencionar que se requiere un análisis factorial de la estructura de la escala para confirmar la validez. Dado el propósito evaluativo de la escala, también es necesario evaluar la sensibilidad al cambio, lo cual los autores abocarán en un futuro estudio. 
En conclusión, la versión adaptada al contexto colombiano del ISA-6 puede considerarse un cuestionario de evaluación de la gravedad de la adicción de los pacientes que asisten a tratamiento. Se demostró que las subescalas que lo componen tienen una buena confiabilidad y la validez de constructo necesarias para introducir su uso en la práctica clínica e individualizar el tratamiento en función de las necesidades de cada paciente.

\section{Agradecimientos}

Agradecemos a todos los pacientes que participaron en el estudio, y al personal administrativo y clínico de las instituciones participantes.

\section{Referencias}

1. Sussman S, Sussman AN. Considering the definition of addiction. Int J Environ Res Public Health. 2011;8:4025-38. https://doi.org/10.3390/ijerph8104025

2. United Nations Office on Drugs and Crime. World Drug Report, 2017. Fecha de consulta: 12 de octubre de 2018. New York: United Nations Office on Drugs and Crime; 2017. p. 68. Disponible en: https://www.unodc.org/wdr2017/field/Booklet_2_HEALTH.pdf

3. Ministerio de Justicia y del Derecho, Ministerio de Salud y Protección Social. Estudio Nacional de Consumo de Sustancias Psicoactivas en Colombia - 2013. Fecha de consulta: 12 de octubre de 2018. Bogotá: ALVI Impresores S.A.S; 2014. Disponible en: https://www.unodc.org/documents/colombia/2014/Julio/Estudio_de_Consumo_UNODC.pdf

4. Leshner Al. Addition is a brain disease, and it matters. Science. 1997;278:45-7. https://doi.org/10.1126/science.278.5335.45

5. French MT, McGeary KA, Chitwood DD, McCoy CB. Chronic illicit drug use, health services utilization and the cost of medical care. Soc Sci Med. 2000;50:1703-13. https://doi.org/10.1016/S0277-9536(99)00411-6

6. Degenhardt L, Whiteford HA, Ferrari AJ, Baxter AJ, Charlson FJ, Hall WD, et al. Global burden of disease attributable to illicit drug use and dependence: Findings from the Global Burden of Disease Study 2010. Lancet. 2013;382:1564-74. https://doi.org/10.1016/S0140-6736(13)61530-5

7. Sartor R. The social impact of drug abuse on community life. Med Law. 1991;10:205-8.

8. Choenni V, Hammink A, van de Mheen D. Association between substance use and the perpetration of family violence in industrialized countries: A systematic review. Trauma Violence Abuse. 2015;18:37-50. https://doi.org/10.1177/1524838015589253

9. United Nations Office on Drugs and Crime. Treatnet-International Network of Drug Dependence Treatment and Rehabilitation Resource Centres. Fecha de consulta: 17 de septiembre de 2015. Disponible en: https://www.unodc.org/treatment/en/drug-treatmentnetwork---home-archive.html

10. United Nations Office on Drugs and Crime. Treatnet. Quality standards for drug dependence treatment and care services. Vienna: United Nations; 2012. Fecha de consulta: 17 de septiembre de 2015. Disponible en:

https://www.unodc.org/docs/treatment/treatnet quality standards.pdf

11. Cacciola JS, Alterman AI, Habing B, Mclellan AT. Recent status scores for version 6 of the Addiction Severity Index (ASI-6). Addiction. 2011;106:1588-602.

https://doi.org/10.1111/j.1360-0443.2011.03482.x

12. Kessler F, Cacciola J, Alterman A, Faller S, Souza-Formigoni ML, Cruz MS, et al. Psychometric properties of the sixth version of the Addiction Severity Index (ASI-6) in Brazil. Rev Bras Psiquiatr 2012;34:24-33. https://doi.org/10.1590/S1516-44462012000100006

13. Díaz-Mesa EM, García-Portilla P, Sáiz PA, Bascarán TB, Casares MJ, Fonseca E, et al. Rendimiento psicométrico de la sexta versión del Addiction Severity Index en español (ASI6). Psicothema. 2010;22:513-9.

14. Hospital Mental de Antioquia. Segundo Taller sobre Aplicación de Instrumentos ClínicoInvestigativos en la Adicción. Manual de aplicación, calificación e interpretación. Bello: Hospital Mental de Antioquia; 1988. p. 147.

15. Martin CS, Langenbucher JW, Chung T, Sher KJ. Truth or consequences in the diagnosis of substance use disorders. Addiction. 2014;109:1773-8. https://doi.org/10.1111/add.12615 
16. McLellan AT. Is addiction an illness—can it be treated? Subst Abus. 2002;23:67-94.

17. Bonett DG. Sample size requirements for testing and estimating coefficient alpha. J Educ Behav Stat. 2002;27:335-40. https://doi.org/10.3102/10769986027004335

18. Walter SD, Eliasziw M, Donner A. Sample size and optimal designs for reliability studies. Stat Med. 1998;17:101-10.

19. Streiner D, Norman G, Cairney J. Health Measurement Scales. A practical guide to their development and use. Fifth Edit. New York: Oxford University Press; 2015.

20. Bonett DG, Wright TA. Sample size requirements for estimating Pearson, Kendall and Spearman correlations. Psychometrika. 2000;65:23-8. https://doi.org/10.1007/BF02294183

21. International Test Commission. International Guidelines on Test Adaptation. Fecha de consulta: 17 de septiembre de 2015. 2005. Disponible en: https:/www.intestcom.org/files/ guideline test_adaptation_2ed.pdf

22. Departamento Administrativo Nacional de Estadística. Clasificación Internacional Uniforme de Ocupaciones. CIUO - 08 A.C. Adaptada para Colombia. Fecha de consulta: 17 de septiembre de 2015. Bogotá D.C.: DANE; 2015. p. 443. Disponible en: https://www.dane. gov.co/files/sen/nomenclatura/ciuo/CIUO_08_AC_2015_07_21.pdf

23. Terwee CB, Bot SD, de Boer MR, van der Windt DA, Knol DL, Dekker J, et al. Quality criteria were proposed for measurement properties of health status questionnaires. J Clin Epidemiol 2007;60:34-42. https://doi.org/10.1016/j.jclinepi.2006.03.012

24. Alterman Al, McDermott PA, Cook TG, Metzger D, Rutherford MJ, Cacciola JS, et al. New scales to assess change in the Addiction Severity Index for the opioid, cocaine, and alcohol dependent. Psychol Addict Behav. 1998;12:233-46. https://doi.org/10.1037/0893-164X.12.4.233

25. Alterman AI, Cacciola JS, Habing B, Lynch KG. Addiction severity index recent and lifetime summary indexes based on nonparametric item response theory methods. Psychol Assess. 2007;19:119-32. https://doi.org/10.1037/1040-3590.19.1.119

26. Cacciola JS, Alterman AI, Habing B, McLellan AT. Recent status scores for version 6 of the Addiction Severity Index (ASI-6). Addiction. 2011;106:1588-602. https://doi.org/10.1111/j.1360-0443.2011.03482.x

27. Grissom GR, Bragg A. Addiction Severity Index: Experience in the field. Int J Addict. 1991;26:55-64.

28. Mokkink LB, Terwee CB, Knol DL, Stratford PW, Alonso J, Patrick DL, et al. The COSMIN checklist for evaluating the methodological quality of studies on measurement properties: $A$ clarification of its content. BMC Med Res Methodol. 2010;10:22. https://doi.org/10.1186/1471-2288-10-22

29. Bastiaens L, Galus J, Goodlin M. The 12 Item W.H.O.D.A.S. as primary self report outcome measure in a correctional community treatment center for dually diagnosed patients. Psychiatr Q. 2015;86:219-24. https://doi.org/10.1007/s11126-014-9322-6

30. Thomas McLellan A, Cacciola JC, Alterman AI, Rikoon SH, Carise D. The Addiction Severity Index at 25: Origins, contributions and transitions. Am J Addict. 2006;15:113-24. https://doi.org/10.1080/10550490500528316

31. Iraurgi-Castillo I. Comparación del instrumento de salud SF-12 frente al SF-36 en pacientes en mantenimiento con metadona. Adicciones. 2007;19:59-67.

32. Lugo LH, García HI, Gómez C. Confiabilidad del cuestionario de calidad de vida en salud SF-36 en Colombia. Rev Fac Nac Salud Pública. 2006;24:37-50.

33. Ramírez-Vélez R, Agredo-Zúñiga RA, Jerez-Valderrama AM. Confiabilidad y valores normativos preliminares del cuestionario de salud SF-12 (Short Form 12 Health Survey) en adultos Colombianos. Rev Salud Pública. 2010;12:807-19.

34. World Health Organization. WHO Disability Assessment Schedule 2.0 (WHODAS 2.0) 2016. Fecha de consulta: 27 de abril de 2016. Disponible en: http://www.who.int/classifications/icf/more whodas/en/

35. Mas-Expósito L, Amador-Campos JA, Gómez-Benito J, Lalucat-Jo L. The World Health Organization Short Disability Assessment Schedule: A validation study in patients with schizophrenia. Compr Psychiatry. 2012;53:208-16. https://doi.org/10.1016/j.comppsych.2011.02.009 
36. Buist-Bouwman MA, Ormel J, De Graaf R, Vilagut G, Alonso J, Van Sonderen E, et al. Psychometric properties of the World Health Organization Disability Assessment Schedule used in the European Study of the Epidemiology of Mental Disorders. Int J Methods Psychiatr Res 2008;17:185-97. https://doi.org/10.1002/mpr.261

37. American Psychiatric Association. Diagnostic and statistical manual of mental disorders. Fifth Edit. Arlington: American Psychiatric Publishing; 2013.

38. Gold LH. DSM-5 and the assessment of functioning: The World Health Organization Disability Assessment Schedule 2.0 (WHODAS 2.0). J Am Acad Psychiatry Law. 2014;42:173-81.

39. World Health Organization. Measuring health and disability: Manual for WHO disability assessment schedule WHODAS 2.0. Üstün T, Kostanjsek N, Chatterji S, Rehm J, editores. Geneve: World Health Organization; 2010.

40. Kiluk BD, Dreifuss JA, Weiss RD, Morgenstern J, Carroll KM. The Short Inventory of Problems - Revised (SIP-R): Psychometric properties within a large, diverse sample of substance use disorder treatment seekers. Psychol Addict Behav. 2013;27:307-14. https://doi.org/1037/a0028445

41. Alterman AI, Cacciola JS, Ivey MA, Habing B, Lynch KG. Reliability and validity of the alcohol short index of problems and a newly constructed drug short index of problems. J Stud Alcohol Drugs. 2009;70:304-7.

42. Kiluk BD, Dreifuss JA, Weiss RD, Horigian VE, Carroll KM. Psychometric properties of a Spanish-language version of the Short Inventory of Problems. Psychol Addict Behav. 2013;27:893-900. https://doi.org/10.1037/a0032805

43. de Vet HCW, Terwee CB, Mokkink LB, Knol DL. Measurement in Medicine. New York: Cambridge University Press; 2011.

44. López-Pina J. Coeficiente alfa y dimensionalidad en los tests psicométricos. Avances en Medición. 2008;6:131-41.

45. Mukaka MM. Statistics corner: A guide to appropriate use of correlation coefficient in medical research. Malawi Med J. 2012;24:69-71.

46. Martínez-Jiménez YP, Cote-Menéndez M, Prieto-Suárez E. Evaluación y diagnóstico multidimensional según el Índice de Severidad de la Adicción, versión 6.0 (ASI-6) en español, de las personas que ingresan a un centro de atención en adicciones en la ciudad de Bogotá, Colombia (2014-2015). Bogotá: Universidad Nacional de Colombia; 2015. Fecha de consulta: 11 de octubre de 2018. Disponible en: http://www.bdigital.unal.edu.co/50808/1/1140814805.2015.pdf

47. Gil HL, Mello DF de, Ferriani M das GC, Silva MA. Perceptions of adolescents students on the consumption of drugs: A case study in Lima, Perú. Rev Lat Am Enfermagem. 2008;16:551-7.

48. Manders WA, Janssens JM, Cook WL, Oud JH, De Bruyn EE, Scholte RH. Perceptions of problem behavior in adolescents' families: Perceiver, target, and family effects. J Youth Adolesc. 2009;38:1328-38. https://doi.org/10.1007/s10964-008-9339-y

49. Mertens JR, Lu YW, Parthasarathy S, Moore C, Weisner CM. Medical and psychiatric conditions of alcohol and drug treatment patients in an HMO. Arch Intern Med. 2003;163:2511-17. https://doi.org/10.1001/archinte.163.20.2511

50. Cullen W, O'Brien S, O'Carroll A, O'Kelly FD, Bury G. Chronic illness and multimorbidity among problem drug users: A comparative cross sectional pilot study in primary care. BMC Fam Pract. 2009;10:25-32. https://doi.org/10.1186/1471-2296-10-25

51. Meier MH, Caspi A, Cerdá M, Hancox RJ, Harrington H, Houts R, et al. Associations between cannabis use and physical health problems in early midlife. JAMA Psychiatry. 2016;73:731-40. https://doi.org/10.1001/jamapsychiatry.2016.0637

52. Richter D, Eikelmann B, Berger K. Use of the SF-36 in the evaluation of a drug detoxification program. Qual Life Res 2004;13:907-14. https://doi.org/10.1023/B:QURE.0000025589.07313.46

53. Neale J. Measuring the health of Scottish drug users. Health Soc Care Community. 2004;12:202-11. https://doi.org/10.1111/j.1365-2524.2004.00489.x

54. Amin-Esmaeili M, Rahimi-Movaghar A, Sharifi V, Hajebi A, Mojtabai R, Radgoodarzi R, et al. Alcohol use disorders in Iran: Prevalence, symptoms, correlates, and comorbidity. Drug Alcohol Depend. 2017;176:48-54. https://doi.org/10.1016/j.drugalcdep.2017.02.018 
55. Subodh BN, Hazari N, Elwadhi D, Basu D. Prevalence of dual diagnosis among clinic attending patients in a de-addiction centre of a tertiary care hospital. Asian J Psychiatr. 2017;25:169-74. https://doi.org/10.1016/j.ajp.2016.10.020

56. Roncero C, Grau-López L, Palma-Álvarez RF, Rodríguez-Cintas L, Ros-Cucurull E, Esojo A, et al. Higher severity of cocaine addiction is associated with tactile and somatic hallucinations. Eur Psychiatry. 2017;42:63-9. https://doi.org/10.1016/j.eurpsy.2016.11.006

57. Østergaard MLD, Nordentoft M, Hjorthøj C. Associations between substance use disorders and suicide or suicide attempts in people with mental illness: A Danish nation-wide, prospective, register-based study of patients diagnosed with schizophrenia, bipolar disorder, unipolar depression or personal. Addiction. 2017;112:1250-9. https://doi.org/10.1111/add.13788

58. Paiva CB, Ferreira IB, Bosa VL, Narváez JC de M. Depression, anxiety, hopelessness and quality of life in users of cocaine/crack in outpatient treatment. Trends Psychiatry Psychother. 2017;39:34-42. https://doi.org/10.1590/2237-6089-2015-0065

59. Henkel D. Unemployment and substance use: A review of the literature (1990-2010). Curr Drug Abuse Rev. 2011;4:4-27. https://doi.org/10.2174/1874473711104010004

60. Janlert U. Unemployment as a disease and diseases of the unemployed. Scand J Work Environ Health. 1997;23 (Suppl. 3):79-83.

61. Velleman R, Bennett G, Miller T, Orford J, Rigby K, Tod A. The families of problem drug users: A study of 50 close relatives. Addiction. 1993;88:1281-9. https://doi.org/10.1111/j.1360-0443.1993.tb02150.x

62. Amin-Esmaeili M, Hefazi M, Radgoodarzi R, Motevalian A, Sharifi V, Hajebi A, et al. Outof-pocket cost of drug abuse consequences: Results from Iranian National Mental Health Survey. East Mediterr Health J. 2017;23:150-60.

63. Wilson IM, Graham K, Taft A. Living the cycle of drinking and violence: A qualitative study of women's experience of alcohol-related intimate partner violence. Drug Alcohol Rev. 2017;36:115-24. https://doi.org/10.1111/dar.12405

64. Gameroff MJ, Wickramaratne P, Weissman MM. Testing the Short and Screener versions of the Social Adjustment Scale - Self-report (SAS-SR). Int J Methods Psychiatr Res. 2012;21:52-65. https://doi.org/10.1002/mpr.358

65. Ray JV, Hall J, Rivera-Hudson N, Poythress NG, Lilienfeld SO, Morano M. The relation between self-reported psychopathic traits and distorted response styles: A meta-analytic review. Personal Disord. 2013;4:1-14. https://doi.org/10.1037/a0026482

66. Busner J, Targum SD, Miller DS. The Clinical Global Impressions scale: Errors in understanding and use. Compr Psychiatry. 2009;50:257-62.

https://doi.org/10.1016/j.comppsych.2008.08.005 